\title{
Energy hypersurface local properties of the $\mathrm{O}_{2} \mathrm{HF}^{-}$rearrangement
}

\author{
G. Dive, D. Dehareng, P. Culot and J.M. Ghuysen \\ Centre d'Ingénierie des Protéines, Université de Liège, Institut de Chimie, B6, 4000 Sart Tilman (Liège I), Belgium
}

Received 29 April 1992

\begin{abstract}
This work analyses a case where.Murrell's proposal, stating that a second-order point must lie above a first-order one, is apparently violated. Study at the SCF-UMP2 level within the D95 + basis set, of one critical point of the $\mathrm{O}_{2} \mathrm{HF}^{-}$anion, previously proposed to be of order two by Lopez, shows the importance of the electronic correlation. The critical point associated with the ${ }^{2} \Pi$ state should rather be considered as a superposition of two first-order ones. The present analysis reveals that this contradistinction can be explained by three unconsidered elements: the local symmetry, the electronic correlation and the internal variables description.
\end{abstract}

\section{Introduction}

In his study of the ion $\mathrm{O}_{2} \mathrm{HF}^{-}$in a $4-31 \mathrm{G}$ basis set [1,2] augmented with $4 \mathrm{sp}$ diffuse functions on each oxygen, Lopez [3] mentioned the existence of a second-order critical point that was $0.835 \mathrm{kcal} / \mathrm{mol}$ lower in energy than another trapped first-order saddle point. These two extrema lie on two different paths connecting two symmetric minima (see fig. 1). Lopez's result is in opposition to Murrell's proposal [4] according to which a minimum energy reaction

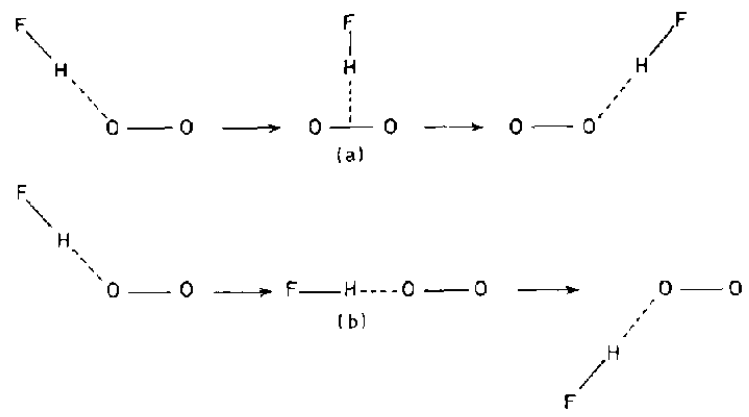

Fig. 1. The two symmetric minima connected by two different reaction paths through either the first-order critical point (a) or the second-order critical point (b).

Correspondence to: $\mathrm{G}$. Dive, Centre d'Ingénierie des Protéines, Université de Liège, Institut de Chimie, B6, 4000 Sart Tilman (Liège 1), Belgium. path cannot pass through a critical point of order higher than 1 . Since the wavefunction for the linear geometry of the second-order point is a ${ }^{2} \Pi$ state, the Renner-Teller effect, i.e. the splitting of the two degenerate electronic states by a coupling with a $\pi$ nuclear vibration [5], is expected. Given that a linear nuclear arrangement characterizes itself by $3 N-5$ and not $3 N-6$ normal modes, the linear symmetry breaking $\pi$ vibrations contain a rotational-like component. The question thus arises as to whether the Hessian eigenvectors are associated with equivalent deformations, one in a given plane and the other in the perpendicular one.

The aim of this work is twofold: first to investigate the variation of the Hessian matrix during a non-linear deformation of the ${ }^{2} \mathrm{II}$ state in order to determine the order of the critical point, and second to study the influence of electronic correlation.

\section{Method of calculation}

All the calculations were performed with GAUSSIAN 86 [6] on a FPS 264 attached to a Digital VAX $11 / 780$.

The geometry optimizations were performed at the full UMP2 level with the Dunning D95 [7] basis set extended with diffuse functions on all the atoms (4 sp on the heavy atoms and $1 \mathrm{~s}$ on the hydrogen), and 
hereafter noted $\mathrm{D} 95++$. The work also includes 4$31 \mathrm{G}+$ calculations for the $\mathrm{C}_{\infty \mathrm{v}}, \mathrm{C}_{\mathrm{s}}$ and $\mathrm{C}_{2 \mathrm{v}}$ conformers.

In the course of the optimization, oscillations between the expected ${ }^{2} \mathrm{~A}_{2}$ state and a close ${ }^{2} \mathrm{~B}_{2}$ state occurred in such a way that the final result was obtained in the ${ }^{2} B_{2}$ state, and not in the ${ }^{2} A_{2}$ state. To solve this problem, a guess for the ${ }^{2} A_{2}$ state was determined by drawing energy curves, in $\mathrm{C}_{2}$ symmetry, for the two ${ }^{2} \mathrm{~A}^{\prime \prime}$ and ${ }^{2} \mathrm{~A}^{\prime}$ states up to the $\mathrm{C}_{2 \mathrm{v}}$ geometry (see figs. 2 and 3 ).

Following the optimizations, frequencies and Hessian analytical calculations were performed in $\mathrm{C}_{\infty \mathrm{v}}$ and $C_{2 v}$ symmetry groups with different numbers of
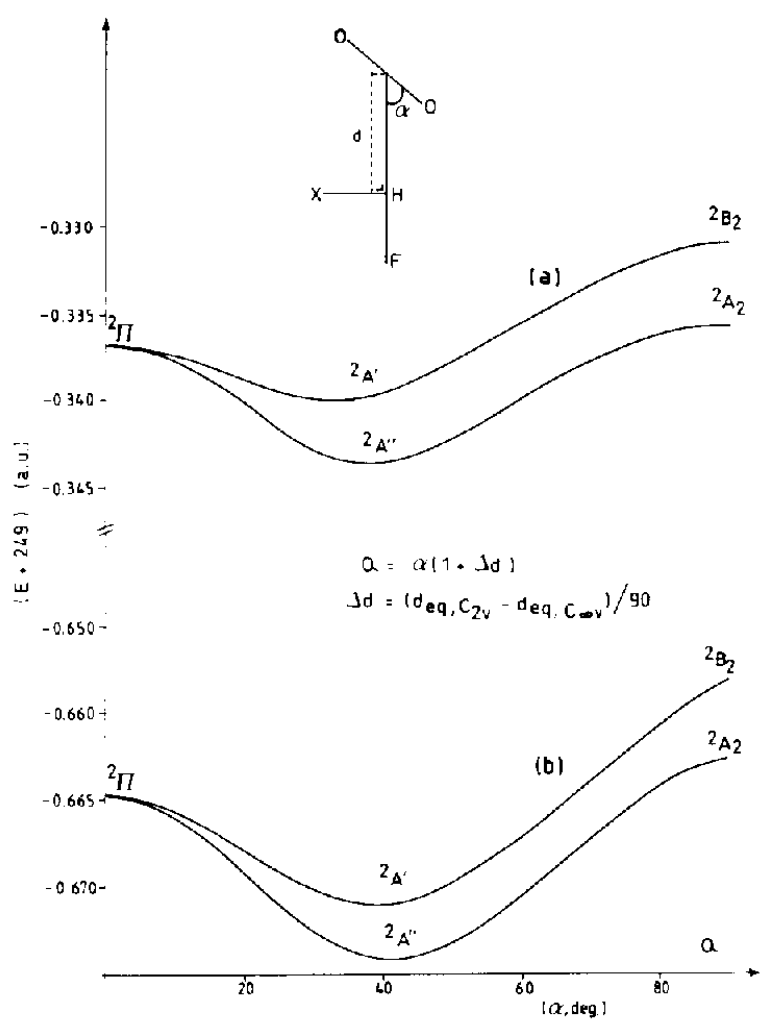

Fig. 2. SCF energy curves in the 4-31G + [3] (a) and D95 ++ (b) basis sets, connecting the ${ }^{2} \Pi$ state to the ${ }^{2} A_{2}$ and ${ }^{2} B_{2}$ states via the ${ }^{2} \mathrm{~A}^{\prime \prime}$ and ${ }^{2} \mathrm{~A}$ ' states. The bond lengths $\mathrm{O}-\mathrm{O}$ and $\mathrm{H}-\mathrm{F}$ are the mean values between the optimized ones for the ${ }^{2} \Pi$ and ${ }^{2} A_{2}$ (in $4-31 \mathrm{G}+$ ) or ${ }^{2} \mathrm{~B}_{2}$ (in $\mathrm{D} 95++$ ) states. The coordinate used to connect $\mathrm{C}_{\infty \mathrm{v}}$ to $\mathrm{C}_{2 \mathrm{v}}$ is a linear combination of the angle $\alpha$ and the distance $d$.

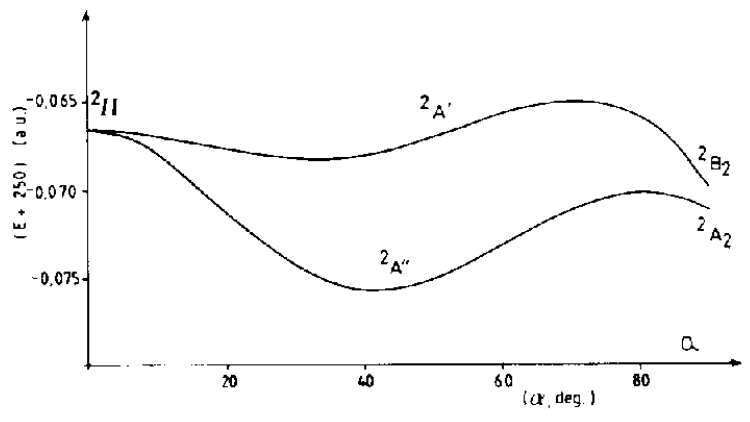

Fig. 3. UMP2 curves within the $\mathrm{D} 95++$ basis set, connecting the ${ }^{2} \Pi$ state to the ${ }^{2} A_{2}$ and ${ }^{2} B_{2}$ states. See legend of fig. 2 .

$$
{ }^{2} \mathrm{~A}_{2} \quad \text { (a) } \quad{ }^{2} \Pi
$$

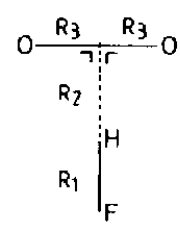

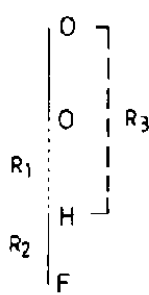

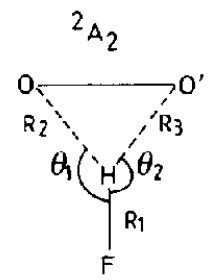

$\varphi_{1}=\mathrm{D}-\mathrm{H}-\mathrm{F}-\mathrm{O}$ torsion

(b)

$$
{ }^{2} \Pi
$$

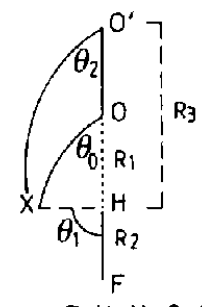

$\varphi_{0}=\mathrm{F}-\mathrm{H}-\mathrm{X}-\mathrm{O}$ torsion

$\varphi_{1}=\mathrm{O}-H-X-F$ torsion

Fig. 4. Definition of the internal coordinates for the ${ }^{2} A_{2}$ and ${ }^{2} \Pi$ electronic states, for the geometry optimization (a) and the frequencies calculation (b).

internal coordinates to emphasize the influence of freezing some symmetry deformations.

\section{Results and discussion}

The geometries of the extrema were optimized with symmetry constraints, using only three internal variables $(R 1, R 2, R 3)$ either for $\mathrm{C}_{2 v}$ or for $\mathrm{C}_{\infty \mathrm{ov}}$ (see fig. $4 a$ ). That the extrema thus obtained (table 1 ) were 
Table 1

Optimized $R 1, R 2, R 3$ values $(\AA)$ for the ${ }^{2} \mathrm{~A}_{2},{ }^{2} \mathrm{~B}_{2}$ and ${ }^{2} \Pi$ electronic states (see fig. 4a), at the SCF and SCF + UMP2 levels, within the $\mathrm{D} 95++$ basis set

\begin{tabular}{|c|c|c|c|c|c|c|}
\hline \multirow[t]{2}{*}{ Variables } & \multicolumn{3}{|c|}{$\mathrm{D} 95++/ /(\mathrm{SCF}+\mathrm{UMP} 2)$} & \multicolumn{3}{|c|}{$\mathrm{D} 95++/ / \mathrm{SCF}$} \\
\hline & ${ }^{2} \mathrm{~A}_{2}$ & ${ }^{2} \mathbf{B}_{2}$ & ${ }^{2} \Pi$ & ${ }^{2} A_{2}$ & ${ }^{2} \mathrm{~B}_{2}$ & ${ }^{2} \Pi$ \\
\hline$R \mathbf{I}$ & 0.98406 & 0.97208 & 1.61788 & 0.94087 & 0.94064 & 1.56332 \\
\hline$R 2$ & 1.74269 & 1.80710 & 0.98163 & 1.80162 & 1.88090 & 0.95348 \\
\hline$R 3$ & 0.74271 & 0.74183 & 2.97628 & 0.67467 & 0.67568 & 2.90569 \\
\hline
\end{tabular}

minima, was confirmed by the three positive eigenvalues of the Hessian.

Although the existence of the imaginary frequencies (one in $\mathrm{C}_{2 v}$, two in $\mathrm{C}_{\infty \mathrm{v}}$ ) is independent of the choice of the internal coordinates, the negative eigenvalues of the Hessian appear only when the additional symmetry breaking internal coordinates are introduced in the molecular description.

\subsection{The critical point of the ${ }^{2} \Pi$ state}

In the linear conformation of the ${ }^{2} \Pi$ state, seven frequencies are calculated (table 2), two of which are imaginary, i.e. correspond to negative eigenvalues of the usual force constants and metric matrices product $F G$ [8]. These two vibrations are the components of a $\pi$ normal mode. The non-equality of the two associated frequencies is due to the fact that the calculation is made with real numbers. Thereby the questions are whether or not the Hessian can correctly reproduce the order of the critical point [9] and whether or not the two imaginary frequencies remain when the symmetry is broken down.

If the $3 N-5$ vibrational prescribed motions (seven variables) are taken into account - i.e. three bond lengths and four angles (valence and torsional) - two negative eigenvalues are always found and the motions represented by their associated eigenvectors correspond to out-linearity deformations. It is already well know that, at a critical point, the number of negative Hessian eigenvalues is independent of the choice of the coordinates in which it is calculated as long as the coordinate set is complete. As an example, we investigated how the number of negative Hessian eigenvalues, at the critical point of the ${ }^{2} \Pi$ state, could be accounted for by different choices (all the possible combinations were envisaged) of only
$3 N-6$ variables. It was found that the number of negative eigenvalues is not always two. There are two cases for which only one negative eigenvalue is found. The suppression of one angle, i.e. of one out-linearity deformation, leads to the disappearance of one $\pi$-like component among the Hessian eigenvectors and thus to the possibility of loosing one negative eigenvalue.

Furthermore, only one imaginary frequency is found for the very nearly linear $\mathrm{C}_{\mathrm{s}}$ conformations or for the $C_{1}$ one of the ${ }^{2} \Pi \rightarrow{ }^{2} A_{2}$ electronic state (table 2 ), thus clearly indicating that the so-called secondorder critical point can be considered as a superposition of two first-order ones. It remains that the description in terms of the Hessian can lead to two negative eigenvalues, depending on the choice on the internal coordinates. This fact is already well known [9-12] that, away from the critical points, the Hessian properties can vary substantially as a function of the coordinates choice.

One point to be noted in table 2 is the following. For the out-equilibrium $\mathrm{C}_{\mathrm{s}}$ and $\mathrm{C}_{\mathbf{1}}$ conformations, the highest frequency $\left(\approx 4000 \mathrm{~cm}^{-1}\right)$ appears to be associated with a deformation mode. This is quite unphysical and is related to the fact that the frequency analysis refers to an unstable nuclear arrangement very near linearity. If one admits that the combination of the two $\pi$ deformations ( $\approx 645$ and $\approx 911 \mathrm{~cm}^{-1}$ ) could be viewed as the composition of one scissoring and one rotation (even though, at the linear conformation, these are not at all rotational modes), one can understand this $\left(\nu_{\text {"rot }} \rightarrow \infty\right)$ discontinuity. 


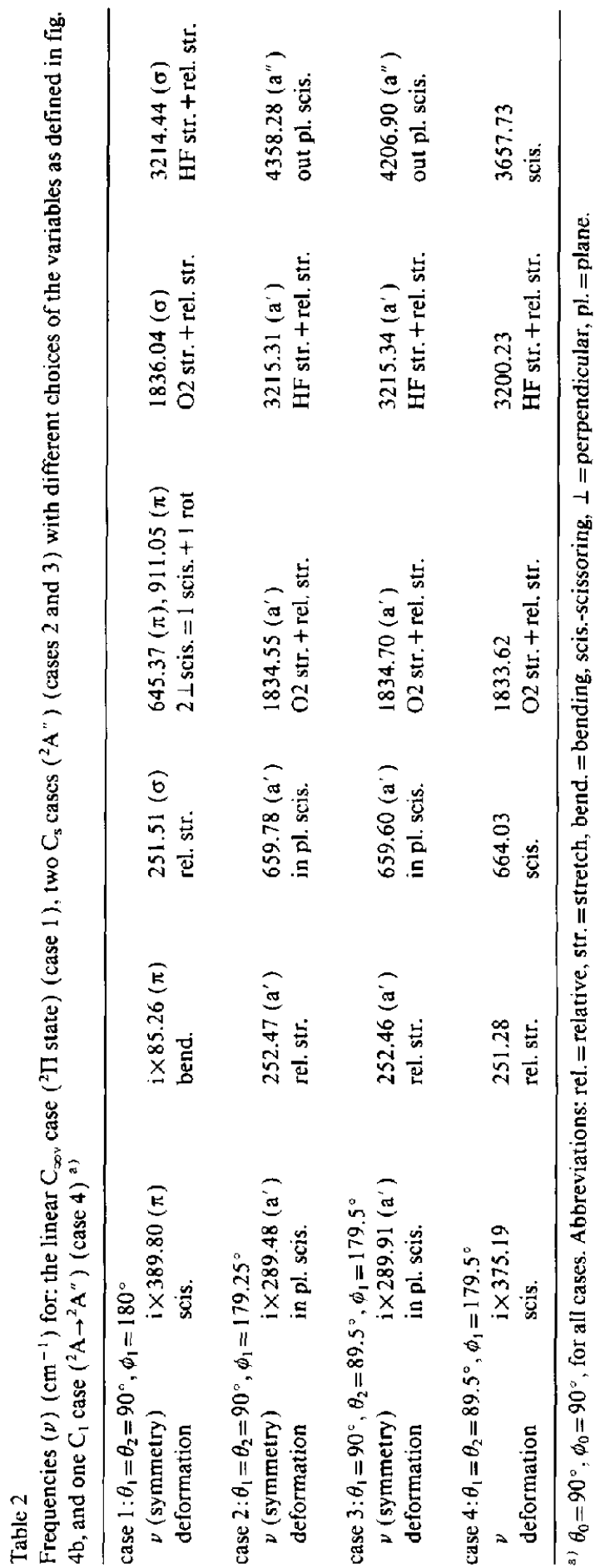

\subsection{Energy curves connecting the ${ }^{2} \Pi$ state to the ${ }^{2} B_{2}$ and ${ }^{2} A_{2}$ states}

At the SCF level (see fig. 2), the 4-31G+ and $\mathrm{D} 95++$ basis sets produce qualitatively similar curves. The ${ }^{2} \mathrm{~B}_{2}$ and ${ }^{2} \mathrm{~A}_{2}$ states are separated by approximately $3 \mathrm{kcal} / \mathrm{mol}$.

The energies for the optimized states are given in table 3. As already stated [3], the ${ }^{2} \Pi$ energy of the so-called second-order point is $0.835 \mathrm{kcal} / \mathrm{mol}$ lower than the energy of the optimized ${ }^{2} \mathrm{~A}_{2}$ first-order point in the 4-31G + basis set. At the SCF level, a similar variation is observed with $\mathrm{D} 95++$, for both the SCF and the SCF+UMP2 optimized geometries. The differences are equal to 1.5 and $9.8 \mathrm{kcal} / \mathrm{mol}$, respectively. In contrast, at the SCF+UMP2 level, within the $\mathrm{D} 95++$ basis set, the ${ }^{2} \Pi$ state lies 10.5 $\mathrm{kcal} / \mathrm{mol}$ higher than the ${ }^{2} \mathrm{~A}_{2}$ state. As seen in figs. 2 and 3 , the correlation is much more important in the $\mathrm{C}_{2 v}$ conformation than in the $\mathrm{C}_{\infty \mathrm{v}}$ one, the difference being around $20 \mathrm{kcal} / \mathrm{mol}$ (table 3 ). It can be calculated straightforwardly from the data of table 1 , that on the average, the two oxygens are nearer $\mathrm{HF}$ in $\mathrm{C}_{2 \mathrm{v}}$ than in $\mathrm{C}_{\text {oov }}$. Hence, it seems obvious that the correlation correction is greater in the former than in the latter symmetry arrangement.

\section{Conclusions}

In the linear conformation of the $\mathrm{O}_{2} \mathrm{HF}^{-}$ion, the two imaginary frequencies that define a second-order critical point are due to the symmetry degeneration. This statement is highlighted by the occurrence of a single imaginary frequency in the nearly linear $\mathrm{C}_{\mathrm{s}}$ and $\mathrm{C}_{1}$ geometries. Under this condition, the ${ }^{2} \Pi$ state should be considered rather as a superposition of two first-order saddle points.

The energies associated with the optimized ${ }^{2} \Pi$ and ${ }^{2} \mathrm{~A}_{2}$ states clearly emphasize the importance of the electronic correlation. At the SCF level, the ${ }^{2} \Pi$ state is lower in energy than the ${ }^{2} \mathrm{~A}_{2}$ state whereas this feature is inverted at the UMP2 level.

\section{Acknowledgement}

This work was supported, in part, by the Belgian program on Interuniversity Poles of Attraction initiated by the Belgian State, Prime Minister's Office, 
Table 3

Energies (hartree) of the ${ }^{2} \Pi,{ }^{2} \mathrm{~A}_{2}$ and ${ }^{2} \mathrm{~B}_{2}$ states for the optimized geometries in the D95 ++ basis set, at the SCF + UMP2 level, and in the 4-31G+ basis set at the SCF level

\begin{tabular}{|c|c|c|c|c|}
\hline \multirow{2}{*}{$\begin{array}{l}4-31 G+[3] \\
S C F \\
4-31 G+/ / S C F\end{array}$} & \multicolumn{4}{|l|}{ D95 ++} \\
\hline & $\begin{array}{l}\mathrm{SCF} \\
\mathrm{D} 95++/ / \mathrm{SCF}\end{array}$ & $\begin{array}{l}\mathrm{SCF}+\mathrm{UMP} 2 \\
\mathrm{D} 95++/ /(\mathrm{SCF}+\mathrm{UMP} 2)\end{array}$ & $\begin{array}{l}\mathrm{SCF} \\
\mathrm{D} 95++/ /(\mathrm{SCF}+\mathrm{UMP} 2)\end{array}$ & $\begin{array}{l}\text { correlation correction a) } \\
\text { D95++// } \\
\text { (SCF+UMP2) }\end{array}$ \\
\hline-249.3368104 & -249.6637268 & -250.0728007 & -249.6624643 & -257.5 \\
\hline-249.3354796 & -249.6612588 & -250.0895302 & -249.6467892 & -277.8 \\
\hline-249.3308317 & -249.6570084 & -250.0823325 & -249.6440237 & -275.0 \\
\hline
\end{tabular}

a) In $\mathrm{kcal} / \mathrm{mol}$.

Science Policy Programming (PAI No. 19), an Action Concertée with the Belgium Government (convention 86/91-90), the Fonds de la Recherche Scientifique Médicale (contract No. 3.4537.88), and a Convention Tripartite between the Région Wallonne, SmithKline Beecham, UK, and the University of Liège. GD is chercheur qualifié of the Fonds National de La Recherche Scientifique, Brussels.

\section{Note added}

The Hessian eigenvalues, and their associated deformation characterization, corresponding to table 2 , are available on request.

\section{References}

[1] R. Ditchfield, W.J. Hehre and J.A. Pople, J. Chem. Phys. 54 (1971) 724.
[2] R. Ditchfield, W.J. Hehre and J.A. Pople, J. Chem. Phys. 56 (1972) 2257.

[3] J.P. Lopez, J. Comput. Chem. 10 (1989) 55.

[4] J.N. Murrell and K.J. Laidler, Trans. Faraday Soc. 64 (1968) 371.

[5] M. Desouter-Lecomte, D. Dehareng, B. Leyh-Nihant, M.Th. Praet, A.J. Lorquet and J.C. Lorquet, J. Phys. Chem. 89 (1985) 214.

[6] M.J. Frisch, M. Head-Gordon, H.B. Schlegel, K. Raghavachari, J.S. Binkley, C. Gonzalez, D.J. DeFrees, D.J. Fox, R.A. Whiteside, R. Seeger, C.F. Melius, J. Baker, L.R. Kahn, J.J.P. Stewart, E.M. Fluder, S. Topiol and J.A. Pople, GAUSSIAN 86-88 (Gaussian, Inc., Pittsburgh, PA).

[7] T.H. Dunning and P.J. Hay, in: Modern theoretical chemistry (Plenum Press, New York, 1976).

[8] E.B. Wilson, J.C. Decius and P.C. Cross, in: Molecular vibrations (McGraw-Hill, New York, 1955).

[9] P. Culot, G. Dive, V.H. Nguyen and J.M. Ghuysen, Theoret. Chim. Acta, accepted for publication.

[10] H.B. Schlegel, Theoret. Chim. Acta 66 (1984) 333.

[11] H.B. Schlegel, Advan. Chem. Phys. 67 (1987) 250.

[12] I.N. Levine, in: Quantum mechanics (Prentice-Hall, Englewood Cliffs, NJ, 1991). 